\title{
Omi/HtrA2 pro-apoptotic marker differs in various hepatocellular carcinoma cell lines owing to ped/pea-15 expression level
}

\author{
ZONGQUAN XU ${ }^{1,2}$, YU CHEN ${ }^{1}$, GUOHUI XU ${ }^{1}$, CHENG PENG $^{2}$, \\ ENYU LIU ${ }^{2}$, YUNGUANG $\mathrm{LI}^{2}$, $\mathrm{JUN} \mathrm{NIU}^{2}$ and $\mathrm{CHANGHAI} \mathrm{LI}^{3}$ \\ ${ }^{1}$ Deparment of Hepatic Oncology, Jiangxi Provincial Cancer Hospital, Nanchang 330029; \\ ${ }^{2}$ Department of Hepatobiliary Surgery, Qilu Hospital, Shandong University, Jinan 250012; \\ ${ }^{3}$ Hepatic Surgery Center Affiliated Tongji Hospital, Tongji Medical College of HuaZhong \\ University of Science and Technology, Wuhan 430030, P.R. China
}

Received August 7, 2014; Accepted November 8, 2014

DOI: $10.3892 /$ or.2014.3656

\begin{abstract}
Omi/HtrA2 promotes cell apoptosis in human cancer cells. Early studies showed that primary hepatocellular carcinoma requires Omi/HtrA2 expression for cell apoptosis. Additionally, the Omi/HtrA2 pro-apoptotic marker demonstrated a difference in some cell types. However, how the Omi/HtrA2 pro-apoptotic marker reacts during the process of hepatocellular carcinoma cell apoptosis remains to be determined. Thus, we investigated the role and possible mechanism of Omi/HtrA2 on hepatocellular carcinoma cell apoptosis using various hepatocellular carcinoma cell lines. The results were analyzed using RT-qPCR and western blot analysis. In the present study, we found that Omi/HtrA2 was overexpressed in hepatocellular carcinoma cell lines and induced hepatocellular carcinoma cell apoptosis. Additiionally, the only manner in which Omi/HtrA2 participated in cell death in PLC cells may be dependent on IAP-binding. Omi/ HtrA2-inducing HepG2 cell apoptosis may mainly depend on its serine protease activity while both IAP-binding and its serine protease activity participated in Hep3B cell apoptosis. This result suggested that $\mathrm{Omi} / \mathrm{HtrA} 2$ pro-apoptotic marker differs in various hepatocellular carcinoma cell lines. PLC cells were also devoid of the expression of ped/pea-15 as the substrate of Omi/HtrA2 serine protease while ped/pea-15 was overexpressed in HepG2 and Hep3B cells and ped/pea-15 expression was higher in HepG2 cells than that in Hep3B cells. These results showed that Omi/HtrA2 overexpression promotes hepatocellular carcinoma cell apoptosis and the ped/pea-15 expression level causes this difference of the Omi/ HtrA2 pro-apoptotic marker in the various hepatocellular carcinoma cell lines.
\end{abstract}

Correspondence to: Dr Changhai Li, Hepatic Surgery Center Affiliated Tongji Hospital, Tongji Medical College of HuaZhong University of Science and Technology, Wuhan 430030, P.R. China E-mail: lichanghai1975@163.com

Key words: Omi/HtrA2 pro-apoptotic manner, hepatocellular carcinoma cell line, ped/pea-15

\section{Introduction}

A correlation between regulation of apoptosis and a number of human pathologies, such as cancer, autoimmune diseases and neurodegenerative disorders has been identified $(1,2)$. Alterations of apoptosis are important in the development of hepatocellular carcinoma cells and many pro- or anti-apoptotic factors are involved in this process.

$\mathrm{Omi} / \mathrm{HtrA} 2$ is a mitochondrial serine protease that is released into the cytoplasm by apoptotic stimuli and induces cell death through its own serine protease activity in addition to attenuating the activity of inhibitor of apoptosis proteins (IAPs). In the cytoplasm, the released mature Omi/HtrA2 binds to and blocks the activity of IAPs, leading to the activation of caspases and execution of cell death. In addition to this caspase-dependent pathway, the cytosolic mature Omi/HtrA2 can induce cell death in a caspase-independent manner that is exclusively dependent on its ability to function as a protease even when caspases are inactivated (3-11).

Previous studies have described Omi/HtrA2 mRNA expression in cancer cells (12-18). Results of previous studies showed that primary hepatocellular carcinoma requires Omi/HtrA2 expression for cell apoptosis (19). It has been reported that the Omi/HtrA2 pro-apoptotic marker differs in some cell types $(20,21)$. However, the manner in which the Omi/HtrA2 pro-apoptotic marker reacts during the process of hepatocellular carcinoma cell apoptosis remains to be determined. Thus, the aim of this study was to investigate the expression status of Omi/HtrA2 in hepatocellular carcinoma cells to predict cell apoptosis alteration and explore the possible mechanism of its effect on hepatocellular carcinoma cell apoptosis. The experimental outcome may provide a theoretical and experimental basis for molecular therapy targeting Omi/HtrA2.

\section{Materials and methods}

Cell culture. Normal L02 hepatocellular and HepG2, Hep3B and PLC hepatocellular carcinoma cells (prepared from the Experimental Center of Hepatobiliary Surgery, Qilu Hospital, Shandong University, Jinan, China) were maintained as monolayers in standard medium comprising Dulbecco's modified 
Eagle's medium (DMEM: $4.5 \mathrm{~g} / \mathrm{l}$ of glucose) containing $10 \%$ heat-inactivated fetal calf serum and supplemented with $20 \mathrm{mM}$ HEPES, $100 \mu \mathrm{g} / \mathrm{ml}$ penicillin and $100 \mu \mathrm{g} / \mathrm{ml}$ streptomycin (Merck, Darmstadt, Germany). The cells were incubated at $37^{\circ} \mathrm{C}, 5 \% \mathrm{CO}_{2}$ and saturated humidity.

Omi/HtrA2 expression vector pEGFP-Omi and Omi/HtrA2 small-interference RNA expression vector psiRNA-Omi construction. After RNA extraction from hypoxic HepG2 cells, cDNA was synthesized with reverse transcriptase as described in the introduction of cDNA Synthesis kit (Gibco-Life Technologies, Carlsbad, CA, USA). For amplification of human Omi/HtrA2, the primers used were: Omi/HtrA2 sense: 5'-ATATTATAGATCTATGGCTGCGCCGAGGGC-3', antisense: 5'-AGTTAGTCGACTCTTCTGTGACCTCAGGG GTC-3'. The Omi/HtrA2 product was digested with SalI and BglII (Fermentas, Shenzhen, China) and ligated into the SalI and $B g l$ II linearized target vector pEGFP-N1 (Clontech, Shanghai, China) to obtain pEGFP-Omi containing the full-length human Omi/HtrA2. Positive clones were confirmed by restriction analysis as well as sequencing of the insert. Expression of Omi/HtrA2 RNA and protein was detected to determine whether to successfully construct Omi/HtrA2 expression vector $\mathrm{pEGFP-Omi.}$

siRNAs were synthesized and purified by GenePharma, Inc. (Shanghai, China). One specific siRNA against the positions of Omi/HtrA2 open reading frame (795-814) was designed. The sequence of Omi/HtrA2 siRNA was: sense: 5'-TCCCACTGCAG AACACGATCACATTCAAGAGATGTGATCGTGTTCTGC AGTTT-3' and antisense: 5'-CAAAAAACTGCAGAACACG ATCACATCTCTTGAATGTGATCGTGTTCTGCAGT-3'. A scrambled version of Omi/HtrA2 siRNA was synthesized and used as a control siRNA (sense: 5'-TCCCACTGCAGGACTT GCCATGCTTAGCTCGTACGTGAGCTAGGCATTT-3' and antisense: 5'-CAAAAAACTGGGAAATGTACACATCTCTC TTGGCTTAACGTTGTTCTGCAGT-3'). The Omi/HtrA2 siRNA and control siRNA product were digested with $B b s \mathrm{I}$ (Fermentas, Shenzhen, China) and ligated into BbsI linearized target vector psiRNA-Hh1neo (Invitrogen Life Technologies, Carlsbad, CA, USA) to obtain Omi/HtrA2 small-interference RNA expression vector psiRNA-Omi and psiRNA. After expression vector psiRNA and psiRNA-Omi/HtrA2 were transfected into HepG2 cells by Lipofectamine 2000 (Invitrogen, Shanghai, China), the expression of Omi/HtrA2 mRNA and protein was analyzed by A quantitative RT-PCR (RT-qPCR) and western blotting to assess silencing efficiency of Omi/HtrA2 small-interference RNA expression vector psiRNA-Omi/HtrA2.

Quantitative RT-PCR analysis. Total RNA was isolated from normal L02 hepatocellular and HepG2, Hep3B and PLC hepatocellular carcinoma cells using TRIzol reagent (Gibco-Life Technologies). cDNA was synthesized with reverse transcriptase as described in the introduction of the cDNA Synthesis kit (Fermentas).

RT-qPCR analysis was carried out using the SYBR-Green PCRMaster Mix (Applied Biosystems, Foster City, CA, USA) using the Applied Biosystems 7500 RT-PCR system according to the manufacturer's instructions. Omi/HtrA2 primers used were sense: 5'-GGGCAGTGCTGTTGTTGTT-3' and antisense: 5'-GCAGGTGCTGTCTTCTCCA-3', GAPDH sense: 5'-GAC
CCCTTCATTGACCTCAAC-3' and antisense: 5'-CTTCTC CATGGTGGTGAAGA-3'. PCR was carried out at a final volume of $25 \mathrm{ml}$ with a SYBR-Green PCR Master Mix, using $1 \mathrm{ml}$ cDNA and $900 \mathrm{nmol}$ of each primer for the respective genes. Cycling conditions were: $50^{\circ} \mathrm{C}$ for $2 \mathrm{~min}$ and $95^{\circ} \mathrm{C}$ for $10 \mathrm{~min}$, followed by 40 cycles at $95^{\circ} \mathrm{C}$ for $15 \mathrm{sec}$ and $60^{\circ} \mathrm{C}$ for $1 \mathrm{~min}$.

Comparison of Omi/HtrA2 mRNA expression was based on the comparative CT method, where L02 cell Omi/HtrA2 mRNA expression value was 1 and GAPDH expression was $\beta$-actin.

Western blot analysis. Adherent and floating cells were homogenized in radioimmunoprecipitation assay buffer (10 mmol/l Tris-HCl, pH 8.0, $10 \mathrm{mmol} / 1$ EDTA, $0.15 \mathrm{~mol} / 1$ $\mathrm{NaCl}, 1 \%$ NP-40, $0.5 \%$ sodium dodecyl sulphate (SDS), $1 \mathrm{mg} / \mathrm{ml}$ Aprotinin, $1 \mathrm{mmol} / 1$ phenyl methyl sulphonyl fluoride). The protein content was measured using the BCA protein assay reagent and $10 \mu \mathrm{g}$ was electrophoresed on a $12.5 \%$ SDS-PAGE gel under non-reducing conditions. Prior to loading the sample on the gel, the protein loads were equalized and the electrophoresed proteins were transferred to nitrocellulose membranes. An equivalent protein loading for each lane was reconfirmed by staining the nitrocellulose membrane with Ponceau using the $36-\mathrm{kDa}$ GAPDH band present in the supplement as a reference marker. The membranes were then probed with primary polyclonal antibody against Omi/HtrA2, XIAP, ped/pea-15, HAX-1 (Santa Cruz Biotechnology, Santa Cruz, CA, USA), followed by peroxidase-labeled secondary antibodies. Western blot analyses were visualized using the enhanced chemiluminescence detection system according to the manufacturer's instructions.

RNA extraction and RT-PCR analysis. Total mRNA was isolated from hepatocellular carcinoma cells using TRIzol reagent following the manufacturer's instructions. cDNA was synthesized with reverse transcriptase as described in the introduction of the cDNA Synthesis kit. For the amplification of Omi/HtrA2 (978 bp), the primers used were: Omi/HtrA2 sense: 5'-GCCATGATGGTGCATGACCGGTTAG-3' and antisense: 5'-AGTCCGTAAATTGGGGCCATGC-3'. $\beta$-actin (245 bp) sense: 5'-GCATGCTCTATAGGAACGCG-3' and antisense: 5'-CGATGGCAATCCTTACGTAAC-3'. The PCR regimen for $\mathrm{Omi} / \mathrm{HtrA} 2$ and $\beta$-actin involved an initial denaturation step of $94^{\circ} \mathrm{C}$ for $30 \mathrm{sec}$, followed by 30 cycles at $94^{\circ} \mathrm{C}$ for $40 \mathrm{sec}, 55^{\circ} \mathrm{C}$ for $40 \mathrm{sec}$ and $72^{\circ} \mathrm{C}$ for $60 \mathrm{sec}$ on a GeneAmp PCR system 9700 (Perkin-Elmer). The densitometric analysis of PCR products was performed using MAGIAS-1000 software and normalized relative to the $\beta$-actin expression for each sample. The same experiments were performed three times.

Detecting the effect of Omi/HtrA2 gene silencing by RNA interference on hepatocellular carcinoma cell viability using MTT assay. Cell viability was determined by measuring cell metabolism using the 3-(4, 5-dimethylthiazol-2-yl)-2, 5-diphenyltetrazolium bromide (MTT) assay. In brief, HepG2 cells, HepG2 cells transfected into psiRNA and HepG2 cells transfected into psiRNA-Omi were plated at $5 \times 10^{3}$ cells/well in 96-well tissue culture plates. After treatment with doxorubicin at concentrations of $0,10^{-4}, 10^{-3}, 10^{-2}$ and $10^{-1} \mathrm{~mol} / \mathrm{l}$ for 
$36 \mathrm{~h}$, MTT was added to each well at a final concentration of $5 \mathrm{mg} / \mathrm{ml}$ and the cells were incubated for $4 \mathrm{~h}$ at $37^{\circ} \mathrm{C}$. The medium was then removed and the cells were dissolved with dimethyl sulfoxide (DMSO; Sigma-Aldrich, St. Louis, MO, USA). Absorbance was measured at $570 \mathrm{~nm}$ (referenced to $650 \mathrm{~nm}$ ) in a microplate reader (Bio-Tech Instruments Inc., Winooski, VT, USA).

As described earlier, the effect of Omi/HtrA2 gene silencing by RNA interference on Hep3B and PLC cell viability was determined using an MTT assay.

Detection of the effect of Omi/HtrA2 gene silencing by RNA interference on hepatocellular carcinoma cell apoptosis by flow cytometric analysis. HepG2 cells transfected into psiRNA and HepG2 cells transfected into psiRNA-Omi were incubated with doxorubicin at a concentration of $10^{-5} \mathrm{~mol} / \mathrm{l}$ for $12 \mathrm{~h}$, at which point the control group without doxorubicin was established. Each group of cells was detached by $0.25 \%$ trypsin and washed twice with cold phosphate-buffered saline. The cells were stained with Annexin V-FITC (Sigma) according to the manufacturer's instructions. The cells were analyzed on a FACS Calibur flow cytometer and data analysis was performed with CellQuest software (Becton Dickinson, Franklin Lakes, NJ, USA). Since necrotic cells also exhibited phosphatidylserine due to the loss of membrane integrity, DNA staining with propidium iodide, added to the Annexin V-FITC solution, was used to distinguish necrotic cells from the Annexin V-positive stained cell clusters.

The effect of Omi/HtrA2 serine protease activity on Hep3B and PLC cell apoptosis was detected using an MTT assay.

Detection of the effect of ucf-101 (Omi/HtrA2 protease inhibitor) on hepatocellular carcinoma cell viability using MTT assay. Cell viability was determined by measuring cell metabolism using an MTT assay. Briefly, HepG2 cells were plated at $5 \times 10^{3}$ cells/well in 96-well tissue culture plates. Following exposure to ucf-101 at concentrations of $0,10^{-4}, 10^{-3}, 10^{-2}$ and $10^{-1} \mathrm{~mol} / \mathrm{l}$ ) (Merck) for $36 \mathrm{~h}$, the cells were exposed to doxorubicin at a concentration of $10^{-3} \mathrm{~mol} / \mathrm{l}$, followed by the addition of MTT to each well at a final concentration of $5 \mathrm{mg} / \mathrm{ml}$. The cells were subsequently incubated for $4 \mathrm{~h}$ at $37^{\circ} \mathrm{C}$. The medium was then removed and the cells were dissolved with DMSO. Absorbance was measured at $570 \mathrm{~nm}$ (referenced to $650 \mathrm{~nm}$ ) in a microplate reader (Bio-Tech Instruments).

As described earlier, the effect of ucf-101 on Hep3B and PLC cell viability was determined using an MTT assay.

Detecting the effect of ucf-101 (Omi/HtrA2 protease inhibitor) on cell apoptosis through flow cytometric analysis. HepG2 cells were incubated with doxorubicin and ucf-101 at concentrations of $10^{-3}$ and $10^{-1} \mathrm{~mol} / \mathrm{l}$, respectively, for $12 \mathrm{~h}$, at which point the control group without ucf-101 was established. Each group of cells was detached by $0.25 \%$ trypsin and washed twice with cold phosphate-buffered saline. The cells were stained with Annexin V-FITC (Sigma-Aldrich) according to the manufacturer's instructions. The cells were analyzed on a FACS Calibur flow cytometer and data analysis was performed with CellQuest software (Becton Dickinson). Since necrotic cells also exhibited phosphatidylserine due to the loss of membrane integrity, DNA staining with propidium iodide, added to the Annexin V-FITC solution, was used to distinguish necrotic cells from the Annexin V-positive stained cell clusters.

The effect of ucf-101 on Hep3B and PLC cell apoptosis were detected using an MTT assay.

Detecting the effect of Hep 22 and Hep3B cell viability when cells transfected with Omi/HtrA2 expression vector pEGFP-Omi were exposed to ucf-101. Cell viability was determined by measuring cell metabolism MTT assay. Briefly, HepG2 cells, HepG2 cells transfected into pEGFP and HepG2 cells transfected into pEGFP-Omi were plated at $5 \times 10^{3}$ cells/well in 96-well tissue culture plates. Following treatment with doxorubicin at a concentration of $0,10^{-4}, 10^{-3}$, $10^{-2}$ and $10^{-1} \mathrm{~mol} / \mathrm{l}$ for $36 \mathrm{~h}$, MTT was added to each well at a final concentration of $5 \mathrm{mg} / \mathrm{ml}$ and the cells were incubated for $4 \mathrm{~h}$ at $37^{\circ} \mathrm{C}$. The medium was then removed and the cells were dissolved with (DMSO; Sigma-Aldrich). Absorbance was measured at $570 \mathrm{~nm}$ (referenced to $650 \mathrm{~nm}$ ) in a microplate reader (Bio-Tech Instruments).

After HepG2 cells, HepG2 cells transfected into pEGFP and HepG2 cells transfected into pEGFP-Omi were exposed to doxorubicin at concentrations of $0,10^{-4}, 10^{-3}, 10^{-2}$ and $10^{-1} \mathrm{~mol} / \mathrm{l}$ ) for $36 \mathrm{~h}$ and the cells were exposed to ucf-101 at a concentration of $10^{-1} \mathrm{~mol} / \mathrm{l}$, the above experiment was repeated.

The effect of Hep3B cell viability when cells transfected with Omi/HtrA2 expression vector pEGFP-Omi were exposed to ucf-101 was determined using an MTT assay.

Detecting the protein expression of XIAP, ped/pea-15 and HAX-1 in HepG2 and Hep3B. HepG2, Hep3B and PLC cells were divided into the control group, the group treated with ucf-101, and the group transfected into pEGFP-Omi. The control groups were incubated with doxorubicin at a concentration of $10^{-3} \mathrm{~mol} / 1$ for $24 \mathrm{~h}$, the groups treated with ucf-101 were cultured at a concentration of $10^{-3} \mathrm{~mol} / \mathrm{l}$ and ucf-101 at $10^{-1} \mathrm{~mol} / \mathrm{l}$ for $24 \mathrm{~h}$, and the groups transfected into pEGFP-Omi were incubated with docxorubicin at a concentration of $10^{-3} \mathrm{~mol} / \mathrm{l}$ for $24 \mathrm{~h}$. The protein expression of XIAP, ped/pea-15 and HAX-1 was detected by western blotting.

Statistical analysis. Values of each target gene were presented as the mean $\pm \mathrm{SD}$. Silencing efficiency of psiRNA-Omi/ HtrA2 in HepG2 cells and the effect of Omi/HtrA2 RNA interference on HepG2 cell apoptosis were evaluated using the paired-samples t-test and subsequent statistical analysis was carried out using one-way ANOVA. Statistical analyses were carried out using SSPS 13.0 for Windows. $\mathrm{P}<0.05$ was considered significant.

\section{Results}

Successful construction of Omi/HtrA2 expression vector pEGFP-Omi and Omi/HtrA2 small-interference RNA expression vector psiRNA-Omi. After HepG2 cells were transfected into pEGFP-Omi, Omi/HtrA2 mRNA and protein expression in HepG2 cells was effectively increased (Fig. 1A, P<0.05), whereas no change was observed for Omi/HtrA2 mRNA and protein expression in HepG2 cells transfected into pEGFP.

After HepG2 cells were transfected into psiRNA-Omi, Omi/HtrA 2 mRNA and protein expression in HepG2 cells was 

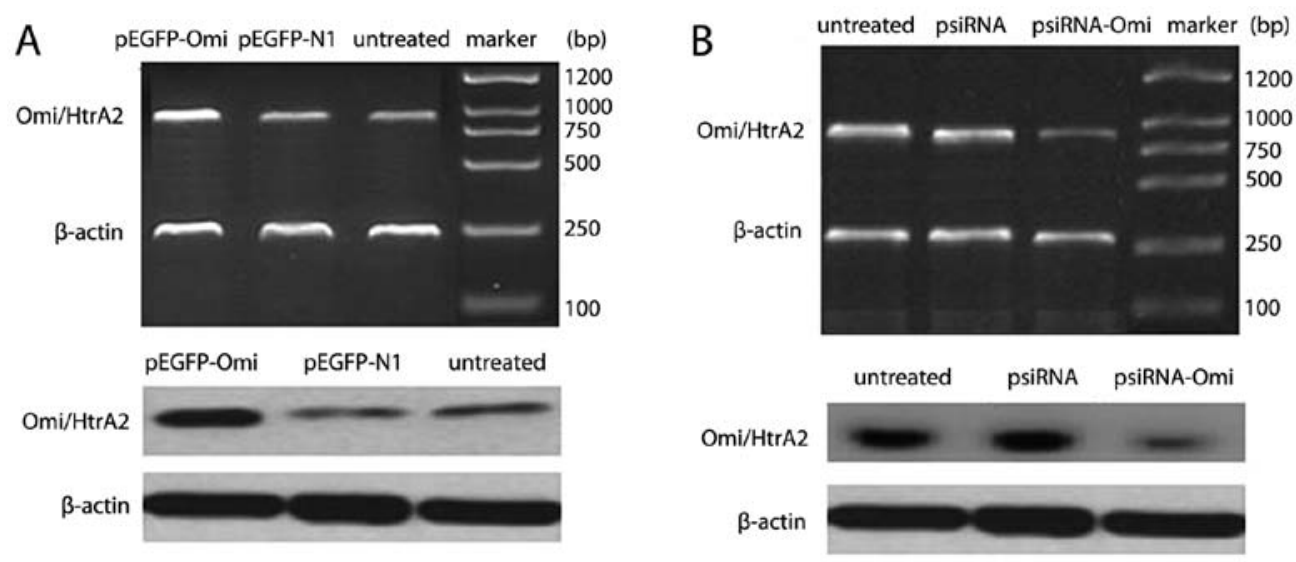

Figure 1. Omi/HtrA2 mRNA and protein expression in HepG2 transfected with Omi/HtrA2 expression vector pEGFP-Omi or Omi/HtrA2 small interference RNA expression vector psiRNA-Omi

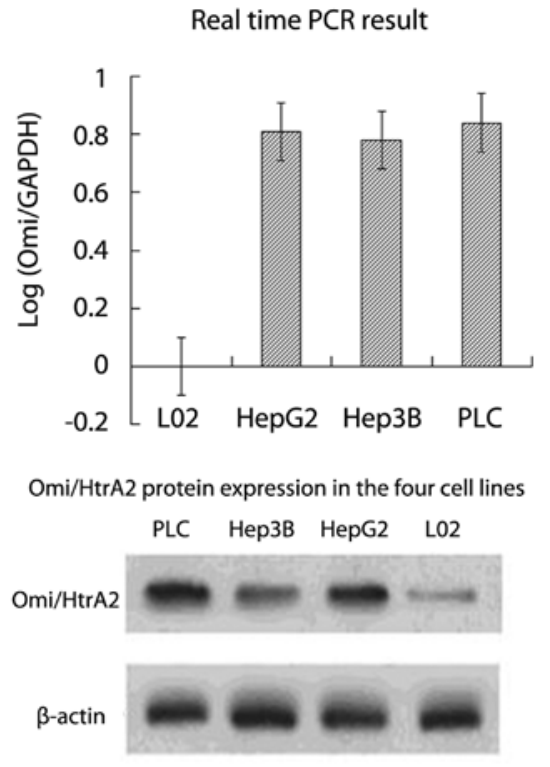

Figure 2. Omi/HtrA2 mRNA and protein expression in normal hepatocellular cell L02 and hepatocellular carcinoma cell lines.

effectively suppressed (Fig. 1B, P<0.05), whereas no change was observed for Omi/HtrA2 mRNA and protein expression in HepG2 cells transfected into psiRNA.

Omi/HtrA2 mRNA and protein overexpress in hepatocellular carcinoma cells. The RT-qPCR assay showed that Omi/HtrA2 mRNA expression level in the HepG2, Hep3B and PLC hepatocellular carcinoma cells was $\sim 8$ times that of the normal L02 hepatocellular cells (Fig. 2, P<0.01). However, no significant difference was identified for the Omi/HtrA2 mRNA expression level for the HepG2, Hep3B and PLC hepatocellular carcinoma cells $(\mathrm{P}>0.05)$. Western blot analysis revealed that the Omi/HtrA2 protein expression was significantly higher in hepatocellular carcinoma cells than that in normal L02 hepatocellular cells (Fig. 2, P<0.05), which was consistent with the result obtained from RT-qPCR.

Effect of silencing of Omi/HtrA2 expression on hepatocellular carcinoma cell viability. MTT assay showed that, after HepG2 cells, HepG2 cells transfected into psiRNA and HepG2 cells transfected into psiRNA-Omi/HtrA2 were incubated with doxorubicin at varying concentrations for $36 \mathrm{~h}$, the cell survival rate of three experimental groups was decreased as the doxorubicin concentration increased. However, the reduced amplitude of HepG2 cells transfected into psiRNA-Omi (RNA interference) was significantly less than that of the other experimental groups (Fig. 3A, $\mathrm{P}<0.05$ ). Similarly, the reduced amplitude of Hep3B (Fig. 3B, P<0.05) and PLC (Fig. 3C, $\mathrm{P}<0.05$ ) cell viability after $\mathrm{Omi} / \mathrm{HtrA} 2$ gene silencing by RNA interference was significantly less than that of the relative experimental groups.

Effect of silencing of Omi/HtrA2 expression on hepatocellular carcinoma cell apoptosis. After HepG2 cells transfected into psiRNA and HepG2 cells transfected into psiRNA-Omi were incubated with doxorubicin at a concentration of $10^{-5} \mathrm{~mol} / 1$ for $12 \mathrm{~h}$, the apoptotic rate of HepG2 cells transfected into psiRNA and HepG2 cells transfected into psiRNA-Omi was 57.8 and $36.9 \%$, respectively (Fig. 4A, $\mathrm{P}<0.05$ ). The result showed that HepG2 cell apoptosis was downregulated after Omi/HtrA2 gene silencing by RNA interference. Similarly, we observed the same effect on Hep3B (Fig. 4B, P <0.05) and PLC (Fig. 4C, P<0.05) cell apoptosis after Omi/HtrA2 gene silencing by RNA interference.

Different effect of ucf-101 (Omi/HtrA2 protease inhibitor)on hepatocellular carcinoma cell viability. MTT assay showed that, after HepG2, Hep3B and PLC cells were incubated with ucf-101 at different concentrations for $36 \mathrm{~h}$, the cell survival rate of HepG2 and Hep3B was increased with the increase in ucf-101 concentration (Fig. 5, P<0.05). The increased amplitude of HepG2 cells was significantly more than that of Hep3B cells, although no change was evident in the cell survival rate of PLC cells.

Different effect of ucf-101 (Omi/HtrA2 protease inhibitor) on hepatocellular carcinoma cell apoptosis. After HepG2 cells were incubated with doxorubicin at a concentration of $10^{-3} \mathrm{~mol} / \mathrm{l}$ and ucf-101 at $10^{-1} \mathrm{~mol} / 1$ for $12 \mathrm{~h}$, the apoptotic rate of HepG2 cells exposed to doxorubicin and ucf-101 and HepG2 cells only exposed to doxorubicin was 35.7 and 58.3\%, 

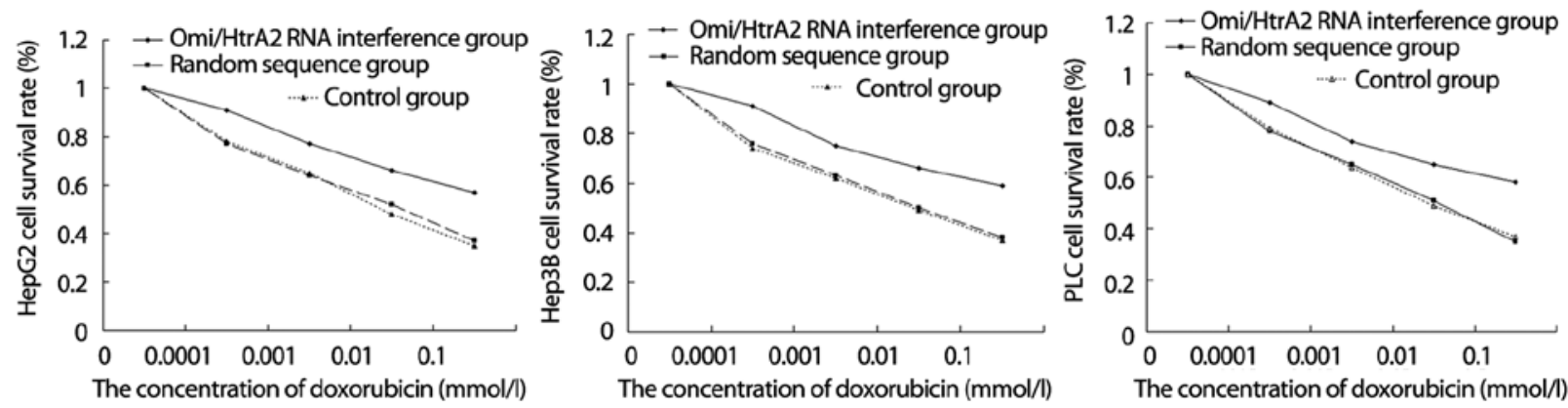

Figure 3. The survival rate of hepatocellular carcinoma cells after Omi/HtrA2 gene silencing by RNA interference.
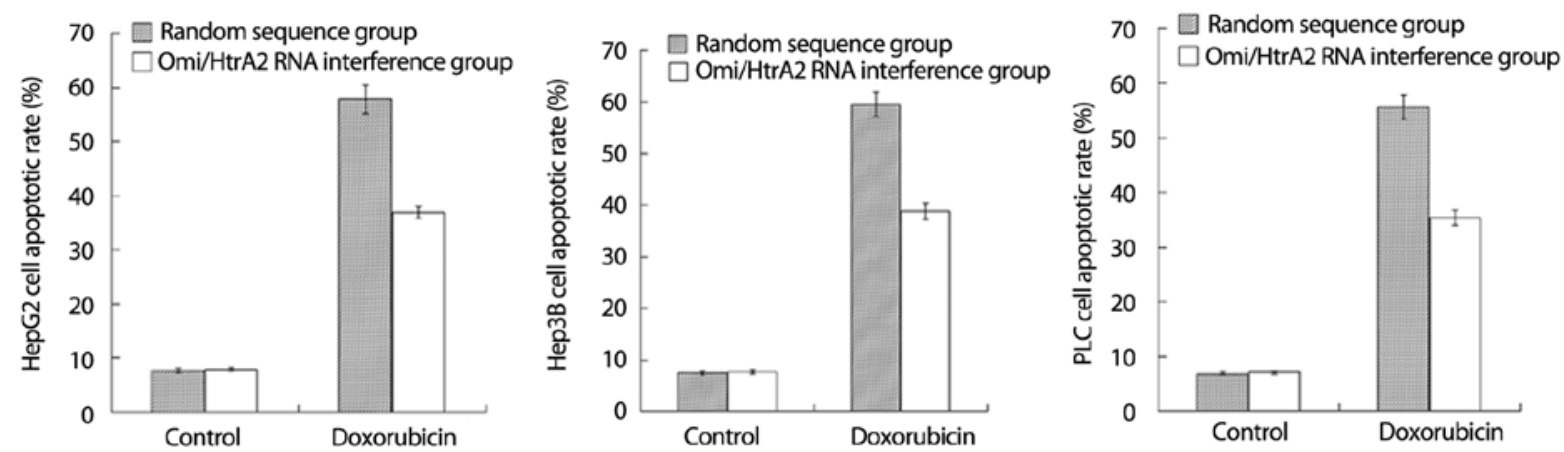

Figure 4. The apoptotic rate of hepatocellular carcinoma cells after Omi/HtrA2 gene silencing by RNA interference.

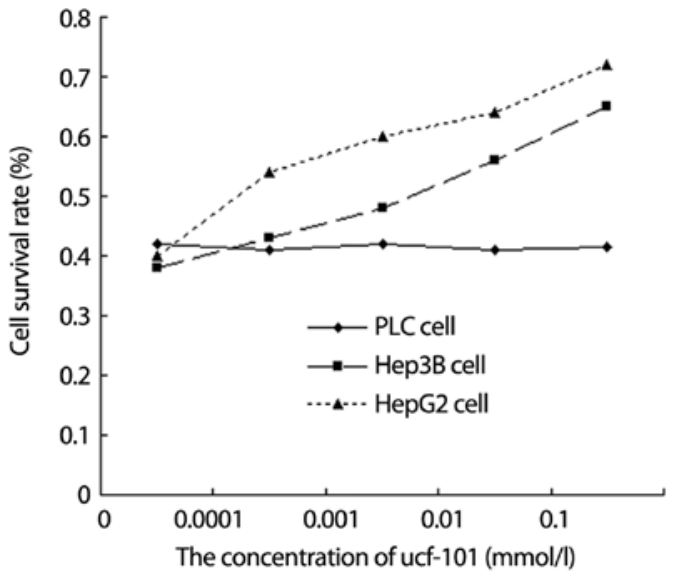

Figure 5. The survival rate of hepatocellular carcinoma cells after exposure to the $\mathrm{Omi} / \mathrm{HtrA} 2$ serine protease inhibitor ucf-101.

respectively (Fig. 6, P<0.05) and that of Hep3B cells exposed to doxorubicin and ucf-101 and Hep3B cells only exposed to doxorubicin was 42.8 and $57.7 \%$, respectively (Fig. 6, $\mathrm{P}<0.05$ ). The result showed that HepG2 and Hep3B cell apoptosis was downregulated following exposure to ucf-101. We also found that PLC cell apoptosis did not change following the exposure of cells to ucf-101.

Effect of HepG2 and Hep3B cell viability when cells transfected with Omi/HtrA2 expression vector pEGFP-Omi were exposed to ucf-101. MTT assay showed that after HepG2, HepG2 transfected into pEGFP-N1 and HepG2 transfected

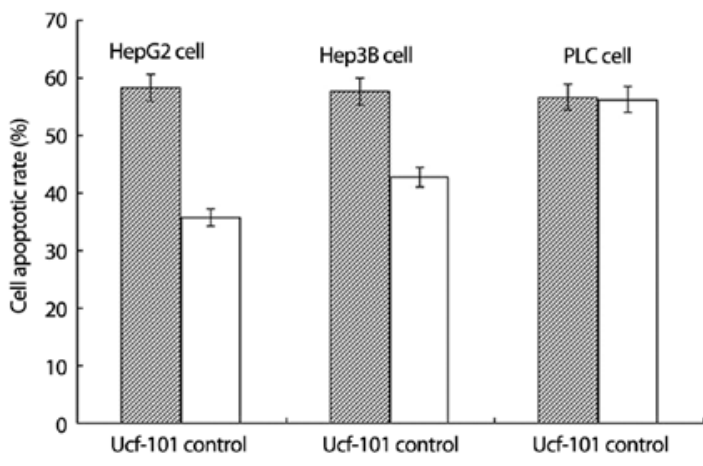

Figure 6. The apoptotic rate of hepatocellular carcinoma cells after exposure to the Omi/HtrA2 serine protease inhibitor ucf-101.

into pEGFP-Omi were incubated under different concentrations of doxorubicin for $36 \mathrm{~h}$, the cell survival rate of the three experimental groups was decreased with an increase in the concentration of doxorubicin. However, the reduced amplitude of HepG2 cells transfected into pEGFP-Omi was significantly more than that of other experimental groups (Fig. 7A, $\mathrm{P}<0.05$ ). When simultaneous treatment with ucf-101 at $10^{-1} \mathrm{~mol} / \mathrm{l}$ was administered to the three cell groups for $36 \mathrm{~h}$, almost no difference was identified in the survival rate of the three groups (Fig. 7B). Compared with the above observation, when Hep3B, Hep3B transfected into pEGFP-N1 and Hep3B transfected into pEGFP-Omi were exposed to doxorubicin under different concentrations, similar results for the change of the survival rate of the three groups were obtained (Fig. 7C, $\mathrm{P}<0.05)$. However, when simultaneous treatment with ucf-101 

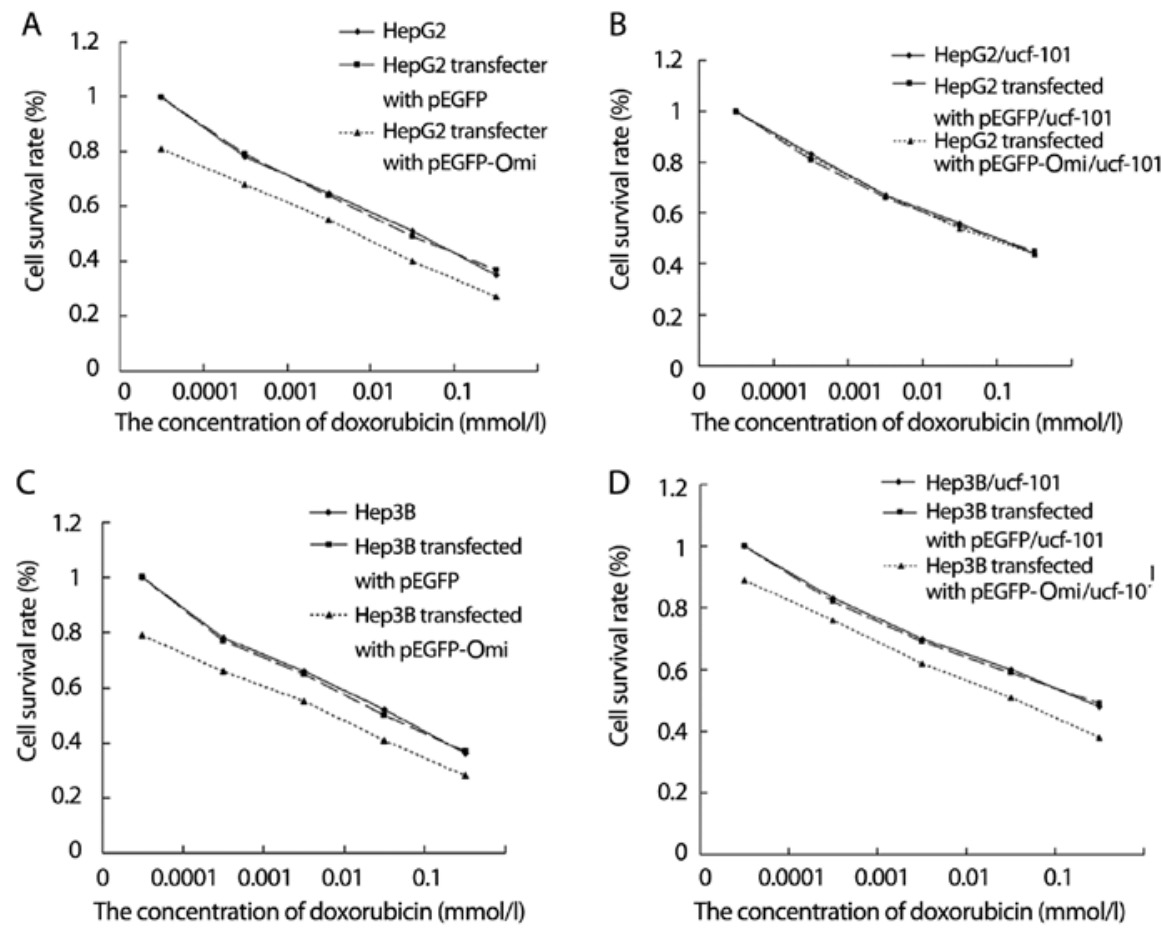

Figure 7. The survival rate of HepG2 and Hep3B cells after cells transfected with Omi/HtrA2 expression vector pEGFP-Omi were exposed to the Omi/HtrA2 serine protease inhibitor ucf-101. The reduced amplitude of HepG2 cells transfected into pEGFP-Omi was significantly more than that of other experimental groups (Fig. 7A, $\mathrm{P}<0.05$ ). The survival rate of the three groups had almost no difference when cells were simultaneously exposed to ucf-101 at $10^{-1} \mathrm{~mol} / 1$ for $36 \mathrm{~h}$ (Fig. 7B). The reduced amplitude of Hep3B cells transfected into pEGFP-Omi was significantly more than that of other experimental groups(Fig. 7C, $\mathrm{P}<0.05$ ). Compared with treatment of cells without ucf-101, the survival rates of the three groups were reduced to a certain degree, but maintained the original difference after simultaneous treatment with ucf-101 at $10^{-1} \mathrm{~mol} / 1$ for $36 \mathrm{~h}$ (Fig. 7D).

at $10^{-1} \mathrm{~mol} / 1$ was administered to the three cell groups for $36 \mathrm{~h}$, the survival rates of the groups increased to a certain degree, but maintained the original difference when cells were not treated with ucf-101 (Fig. 7D). These results suggested that ucf-101 almost offset the effect of Omi/HtrA2 expression vector pEGFP-Omi on HepG2 cell viability. By contrast, for Hep3B cells, the experiment outcome showed that ucf-101 partly counteracted the effect of Omi/HtrA2 expression vector pEGFP-Omi on cell viability.

Protein expression of XIAP, ped/pea-15 and HAX-1 in HepG2, Hep $3 B$ and PLC cells. To clarify the possible mechanism of the difference of Omi/HtrA2 pro-apoptotic marker in various hepatocellular carcinoma cell lines, we detected the protein expression of XIAP, ped/pea-15 and HAX-1 in HepG2, Hep3B and PLC cells. Western blot analysis found that XIAP was overexpressed in HepG2, Hep3B and PLC cells but its expression had no difference among the cell lines, while HAX-1 was not expressed in HepG2, Hep3B and PLC cells. PLC cells were devoid of ped/pea-15 expression while ped/pea-15 was overexpressed in HepG2 and Hep3B cells and ped/pea-15 expression was higher in HepG2 cells than that in Hep3B cells $(\mathrm{P}<0.05)$ (Fig. 8A). To identify the above detection, we examined the protein expression of XIAP, ped/pea-15 and HAX-1 in HepG2, Hep3B and PLC cells treated with ucf-101 or transfected into pEGFP-Omi for $24 \mathrm{~h}$. The results showed no change in the protein expression of XIAP and HAX-1 in HepG2, Hep3B and PLC cells. However, the ped/pea-15 protein expression increased when HepG2 and Hep3B cells were exposed to ucf-101 $(\mathrm{P}<0.05)($ Fig. 8B) but decreased when HepG2 and
Hep3B cells were transfected into pEGFP-Omi $(\mathrm{P}<0.05)$ (Fig. 8C). At the same time, the change of ped/pea-15 protein expression in HepG2 cells was more obvious than that in Hep3B cells (Fig. 8D).

\section{Discussion}

In the present study, we analyzed $\mathrm{Omi} / \mathrm{HtrA} 2$ expression in normal L02 hepatocellular and HepG2, Hep3B and PCL hepatocellular carcinoma cells through RT-qPCR and western blot analysis. The results showed that $\mathrm{Omi} / \mathrm{HtrA} 2$ was overexpressed in the hepatocellular carcinoma cells. Our study also demonstrated that hepatocellular carcinoma cell apoptosis was downregulated after Omi/HtrA2 gene silencing by RNA interference and induced when cells were transfected with Omi/HtrA2 expression vector pEGFP-Omi. Thus, we suggest that $\mathrm{Omi} / \mathrm{HtrA} 2$ overexpression promotes hepatocellular carcinoma cell apoptosis. We previously reported that Omi/HtrA2 was overexpressed in hepatocellular carcinoma tissues and Omi/HtrA2 expression was closely correlated with tumor size, tumor differentiation, clinical stage and lymph-node metastasis (19). The present finding that $\mathrm{Omi} / \mathrm{HtrA} 2$ induces hepatocellular carcinoma cell apoptosis confirmed and extended the observations made in our previous study.

The serine protease $\mathrm{Omi} / \mathrm{HtrA} 2$ is released from mitochondria into the cytosol in response to apoptotic stimuli, inducing cell death in a caspase-dependent manner by interacting with the IAP as well as in a caspase-independent manner that relies on its protease activity. Omi/HtrA2 proapoptotic function presents a 'dual' nature of Omi/HtrA2 proapoptotic activity, 

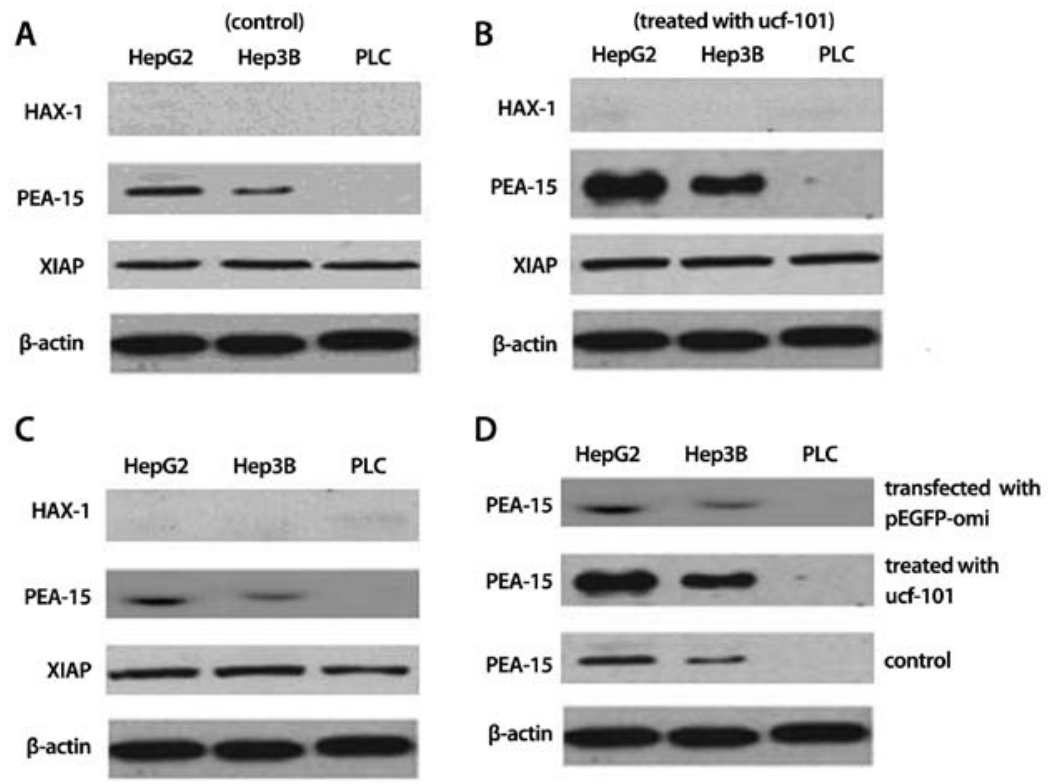

Figure 8. Protein expression of XIAP, ped/pea-15 (PEA-15) and HAX-1 in HepG2, Hep3B and PLC cells. XIAP overexpressed in HepG2, Hep3B and PLC cells and its expression had no difference among them and HepG2, Hep3B and PLC cells had no HAX-1 expression. (A) PLC cells were devoid of ped/pea-15 expression although ped/pea-15 was overexpressed in HepG2 and Hep3B cells. However, ped/pea-15 expression was higher in HepG2 cells than that in Hep3B cells. (B) The protein expression of XIAP and HAX-1 in HepG2, Hep3B and PLC cells showed no change, whereas ped/pea-15 protein expression increased in HepG2 and Hep3B cells following cell exposure to ucf-101. (C) The protein expression of XIAP and HAX-1 in HepG2, Hep3B and PLC cells did not change, whereas the ped/pea-15 protein expression decreased in HepG2 and Hep3B cells when cells were transfected into pEGFP-Omi. (D) The change of ped/pea-15 protein expression in HepG2 cells was more evident than that in Hep3B cells when transfected into pEGFP-Omi or exposed to ucf-101.

which may be dependent on IAP binding or on the serine protease properties.

Previous studies have demonstrated that the manner in which (for IAP-binding or serine protease activity) Omi/HtrA2 participates in cell death may differ in some cell types. Blink et al (20) found that, for the usual apoptotic process in neutrophil inactivation of IAPs was sufficient for $\mathrm{Omi} / \mathrm{HtrA} 2$ to realize its proapoptotic potential and the serine protease component was not essential, although under certain conditions $\mathrm{Omi} / \mathrm{HtrA} 2$ may mediate cell death through its serine protease properties from within the mitochondria. Srinivasula et al $(20,21)$ found that in 293 cells transfected with the active site mutant $\mathrm{Omi} / \mathrm{HtrA} 2$, which had no protease activity, caspase activation and cell death were markedly reduced, whereas transfection of the wild-type protein induced both events and resulted in a significant reduction in the amount of XIAP in the transfected cells, which appeared to be a substrate of Omi/HtrA2.

The abovementioned studies provided important findings. However, whether the Omi/HtrA2 dual pro-apoptotic marker (the way of IAP-binding or serine protease activity) demonstrates a difference in the various hepatocellular carcinoma cell lines remained to be elucidated. In our experiments, the effect of $\mathrm{Omi} / \mathrm{HtrA} 2$ on hepatocellular carcinoma cell viability and apoptosis through using ucf-101 (Omi/HtrA2 protease inhibitor) was examined. We found that HepG2 and Hep3B cell viability and apoptosis were suppressed by $\mathrm{Omi} / \mathrm{HtrA} 2$ serine protease inhibitor ucf-101 while PLC cell viability and apoptosis was not affected by ucf-101. Omi/HtrA2 serine protease activity was involved in the process of HepG2 and Hep3B cell apoptosis, whereas $\mathrm{Omi} / \mathrm{HtrA} 2$ serine protease activity did not participate in PLC cell apoptosis. Therefore, we suggest that in PLC cells the only manner in which $\mathrm{Omi} / \mathrm{HtrA} 2$ induces cell death may be dependent on IAP-binding under some conditions.

In addition, our study showed that the increased amplitude of HepG2 cell viability was higher than that of Hep3B cells following cell exposure to ucf-101. Thus, Omi/HtrA2 induction of cell apoptosis in HepG2 and Hep3B cells is also different and $\mathrm{Omi} / \mathrm{HtrA} 2$ induction of HepG2 cell apoptosis may mainly be dependent on its serine protease activity while IAP-binding and its serine protease activity participate in Hep3B cell apoptosis. To identify how Omi/HtrA2 induces HepG2 and Hep3B cell apoptosis, we detected the change of HepG2 and Hep3B cell viability when cells transfected with Omi/HtrA2 expression vector pEGFP-Omi were exposed to ucf-101 (Omi/HtrA2 protease inhibitor). Our study results demonstrate that ucf-101 almost offset the effect of Omi/HtrA2 expression vector pEGFP-Omi on HepG2 cell viability. By contrast, for Hep3B cells, the experiment outcome showed that ucf-101 partly counteracted the effect of Omi/HtrA2 expression vector pEGFP-Omi on cell viability. This result confirms the above supposition that the way that Omi/HtrA2 induces HepG2 cell apoptosis may be mainly dependent on Omi/HtrA2 serine protease activity while IAP-binding and its serine protease activity participate in Hep3B cell apoptosis. Based on the above data, we consider that the Omi/HtrA2 marker induces cell apoptosis differently in various hepatocellular carcinoma cell lines under varying conditions.

Of note, which factors cause Omi/HtrA2 pro-apoptotic marker to differ in various hepatocellular carcinoma cell lines remains to be elucidated. Yang et al (22) reported that the overexpression of Omi/HtrA2 causes caspase-independent cell death, depending on the protease activity of Omi/ HtrA2 alone, independent of IAP inhibition, although 
no functional substrates were identified. Subsequently, ped/pea-15 and HAX-1 (the mitochondrial anti-apoptotic protein $\mathrm{HS} 1$-associated protein $\mathrm{X}-1$ ) were identified as Omi/ HtrA2 substrates by Trencia et al (23) and Cilenti et al (24), respectively, and the two substrates were involved in the apoptotic process. Therefore, we postulate that ped/pea-15 and $\mathrm{HAX}-1$ as the substrates of $\mathrm{Omi} / \mathrm{HtrA} 2$ serine protease may be important in the Omi/HtrA2 pro-apoptotic marker difference. Thus, we detected ped/pea-15, HAX-1 and XIAP protein expression in HepG2, Hep3B and PLC cells. The results show that ped/pea-15 protein expression in HepG2, Hep3B and PLC cells has a significant difference. Moreover, PLC cells have no ped/pea-15 protein expression while ped/pea-15 was overexpressed in HepG2 and Hep3B cells and ped/pea-15 protein expression in HepG2 cells was higher than that in Hep3B cells. This outcome provides a reasonable explanation for the above experiment that cell viability and apoptosis of HepG2, Hep3B and PLC cells treated with ucf-101 had an obvious difference. Thus, ped/pea-15 expression level causes the difference of Omi/HtrA2 pro-apoptotic marker in various hepatocellular carcinoma cell lines. Nevertheless, additional experiments are required to validate the findings.

Taken together, our study results show that Omi/HtrA2 overexpression facilitates hepatocellular carcinoma cell apoptosis and that, for IAP-binding (caspase-dependent pathway) or serine protease activity (caspase-independent pathway) of Omi/HtrA2, the manner in which Omi/HtrA2 induces cell apoptosis differs in the various hepatocellular carcinoma cell lines and ped/pea-15 expression level results in this difference of Omi/HtrA2 pro-apoptotic marker. However, the role and possible mechanisms of Omi/HtrA2 expression on hepatocellular carcinoma cell apoptosis may provide a novel option for deploying Omi/HtrA2 to carry out targeting therapy in hepatocellular carcinoma.

\section{Acknowledgements}

This study was supported by the Chinese Foundation for Hepatitis Prevention and Control, TianQing Liver Disease Research Fund (no. TQGB2011019), the Jiangxi Provincial Natural Sciences Foundation Research Grant (no. 20132 BAB205048), the Jiangxi Province Science and Technology Support Program (no. 20122BBG70119), and the National Natural Sciences Foundation Research Grant of China (no. 81460442).

\section{Reference}

1. Kroemer G and Reed JC: Mitochondrial control of cell death. Nat Med 6: 513-519, 2000.

2. Thompson CB: Apoptosis in the pathogenesis and treatment of disease. Science 267: 1456-1462, 1995.

3. Suzuki Y, Imai Y, Nakayama H, Takahashi K, Takio K and Takahashi R: A serine protease, HtrA2, is released from the mitochondria and interacts with XIAP, inducing cell death. Mol Cell 8: 613-621, 2001.

4. Hegde R, Srinivasula SM, Zhang Z, et al: Identification of $\mathrm{Omi} / \mathrm{HtrA} 2$ as a mitochondrial apoptotic serine protease that disrupts inhibitor of apoptosis protein-caspase interaction. J Biol Chem 277: 432-438, 2002.
5. Martins LM, Iaccarino I, Tenev T, et al: The serine protease Omi/HtrA2 regulates apoptosis by binding XIAP through a reaper-like motif. J Biol Chem 277: 439-444, 2002.

6. Verhagen AM, Silke J, Ekert PG, et al: HtrA2 promotes cell death through its serine protease activity and its ability to antagonize inhibitor of apoptosis proteins. J Biol Chem 277: 445-454, 2002.

7. Liu Z, Sun C, Olejniczak ET, et al: Structural basis for binding of Smac/DIABLO to the XIAP BIR3 domain. Nature 408: 1004-1008, 2000.

8. Wu G, Chai J, Suber TL, Wu JW, Du C, X Wang and Shi Y: Structural basis of IAP recognition by Smac/DIABLO. Nature 408: 1008-1012, 2000.

9. Faccio L, Fusco C, Viel A and Zervos AS: Tissue specific splicing of Omi stress-regulated endoprotease leads to an inactive protease with a modified PDZ motif. Genomics 68: 343-347, 2000.

10. Li W, Srinivasula SM, Chai J, et al: Structural insights into the pro-apoptotic function of mitochondrial serine protease HtrA2/Omi. Nat Struct Biol 9: 436-441, 2002.

11. Suzuki Y, Takahashi-Niki K, Akagi T, Hashikawa T and Takahashi R: Mitochondrial protease Omi/HtrA2 enhances caspase activation through multiple pathways. Cell Death Differ 11: 208-216, 2004.

12. Faccio L, Fusco C, Chen A, Martinotti S, Bonventre JV and Zervos AS: Characterization of a novel human serine protease that has extensive homology to bacterial heat shock endoprotease HtrA and is regulated by kidney ischemia. J Biol Chem 275: 2581-2588, 2000.

13. Lee SH, Lee JW, Kim HS, et al: Immunohistochemical analysis of Omi/HtrA2 expression in stomach cancer. APMIS 111: 586-590, 2003.

14. Hu XY, Xu YM, Chen XC, Ping H, Chen ZH and Zeng FQ: Immunohistochemical analysis of $\mathrm{Omi} / \mathrm{HtrA} 2$ expression in prostate cancer and benign prostatic hyperplasia. APMIS 114: 893-898, 2006

15. Wu SJ, Ng LT, Lin DL, Huang SN, Wang SS and Lin CC: Physalis peruviana extract induces apoptosis in human HepG2 cells through CD95/CD95L system and the mitochondrial signaling transduction pathway. Cancer Lett 215: 199-208, 2004.

16. Yamaguchi H, Bhalla K and Wang HG: Bax plays a pivotal role in the apsigargin-induced apoptosis of human colon cancer HCT116 cells by controlling Smac/Diablo and Omi/HtrA2 release from mitochondria. Cancer Res 63: 1483-1489, 2003.

17. Kempkensteffen C, Hinz S, Christoph F, et al: Expression levels of the mitochondrial IAP antagonists Smac/DIABLO and Omi/HtrA2 in clear-cell renal cell carcinomas and their prognostic value. J Cancer Res Clin Oncol 134: 543-550, 2008.

18. Zhou H, Chen J, Lu X, Shen C, Zeng J, Chen L and Pei Z: Melatonin protects against rotenone-induced cell injury via inhibition of Omi and Bax-mediated autophagy in Hela cells. J Pineal Res 52: 120-127, 2012.

19. Xu Z, Chen X, Peng C, Liu E, Li Y, Li C and Niu J: The expression and clinical significance of Omi/Htra2 in hepatocellular carcinoma. Hepatogastroenterology 60: 6-13, 2012.

20. Blink E, Maianski NA, Alnemri ES, Zervos AS, Roos D and Kuijpers TW: Intramitochondrial serine protease activity of $\mathrm{Omi} / \mathrm{HtrA} 2$ is required for caspase-independent cell death of human neutrophils. Cell Death and Differ 11: 937-939, 2004.

21. Srinivasula SM, Gupta S, Datta P, et al: Inhibitor of apoptosis proteins are substrates for the mitochondrial serine protease Omi/HtrA2. J Biol Chem 278: 31469-31472, 2003.

22. Yang QH, Church-Hajduk R, Ren JY, Newton ML and Du CY: $\mathrm{Omi} / \mathrm{HtrA} 2$ catalytic cleavage of inhibitor of apoptosis (IAP) irreversibly inactivates IAPs and facilitates caspase activity in apoptosis. Genes Dev 17: 1487-1496, 2003.

23. Trencia A, Fiory F, Maitan MA, et al: Omi/HtrA2 promotes cell death by binding and degrading the anti-apoptotic protein ped/pea-15. J Biol Chem 279: 46566-46572, 2004.

24. Cilenti L, Soundarapandian MM, Kyriazis GA, et al: Regulation of HAX-1 anti-apoptotic protein by Omi/HtrA2 protease during cell death. J Biol Chem 279: 50295-50301, 2004. 